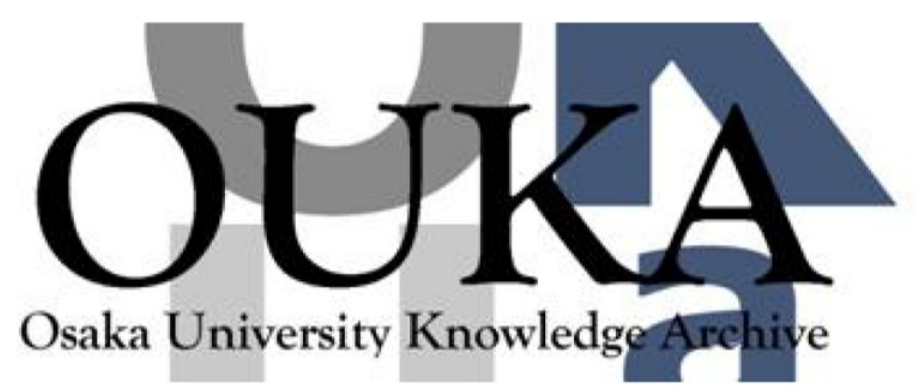

\begin{tabular}{|c|l|}
\hline Title & $\begin{array}{l}\text { Petawatt-laser direct heating of uniformly } \\
\text { imploded deuterated-polystyrene shell target }\end{array}$ \\
\hline Author(s) & $\begin{array}{l}\text { Kitagawa, Yoneyoshi; Sentoku, Yasuhiko; } \\
\text { Akamatsu, Shin et al. }\end{array}$ \\
\hline Citation & Physical Review E. 71(1) p. 016403 \\
\hline Issue Date & $2005-01$ \\
\hline oaire:version & VoR \\
\hline URL & $\begin{array}{l}\text { https://hdl. handle. net/11094/3252 } \\
\text { Kitagawa Yoneyoshi, Sentoku Yasuhiko, Akamatsu } \\
\text { Shin, Sakamoto Wataru, Tanaka Kazuo, Kodama } \\
\text { Ryosuke, Nishimura Hiroaki, Inubushi Yuichi, } \\
\text { Nakai Mitsuo, Watari Takeshi, Norimatsu } \\
\text { Takayoshi, Sunahara Atsushi, Physical Review E, } \\
\text { 71, 1, 016403, 2005-01. "Copyright 2005 by the } \\
\text { American Physical Society." }\end{array}$ \\
\hline rights \\
\hline Note & \begin{tabular}{l} 
\\
\hline
\end{tabular} \\
\hline
\end{tabular}

Osaka University Knowledge Archive : OUKA

https://ir. Library. osaka-u. ac. jp/

Osaka University 


\title{
Petawatt-laser direct heating of uniformly imploded deuterated-polystyrene shell target
}

\author{
Yoneyoshi Kitagawa, ${ }^{1 *}$ Yasuhiko Sentoku, ${ }^{2}$ Shin Akamatsu, ${ }^{1}$ Wataru Sakamoto, ${ }^{1}$ Kazuo A. Tanaka, ${ }^{1}$ Ryosuke Kodama, ${ }^{1}$ \\ Hiroaki Nishimura, ${ }^{1}$ Yuichi Inubushi, ${ }^{1}$ Mitsuo Nakai, ${ }^{1}$ Takeshi Watari, ${ }^{1}$ Takayoshi Norimatsu, ${ }^{1}$ and Atsushi Sunahara ${ }^{1}$ \\ ${ }^{1}$ Institute of Laser Engineering, Osaka University, Suita, Osaka 565-0871, Japan \\ ${ }^{2}$ Department of Physics, University of Nevada, Reno, Nevada 89506, USA
}

(Received 13 February 2004; published 7 January 2005)

\begin{abstract}
A uniformly imploded deuterated polystyrene (CD) shell target is fast-heated by a Petawatt (PW) laser without cone guide. The best illumination timing is found to be in a narrow region around $80 \pm 20$ picoseconds from the onset of the stagnation phase, where thermal neutrons are enhanced four to five times by the PW laser of energy less than $10 \%$ of the implosion laser. The timing agrees with the timings of enhancement of the $\mathrm{x}$-ray emission from the core and reduction of the bremsstrahlung radiation from scattered hot electrons. The PW laser, focused to the critical density point, generates the energetic electrons within as narrow an angle as $30^{\circ}$, which then heats the imploded CD shell to enhance thermal neutrons. These results first demonstrate that the PW laser directly heats the imploded core without any conelike laser guide.
\end{abstract}

DOI: 10.1103/PhysRevE.71.016403

PACS number(s): $52.38 . \mathrm{Kd}, 41.75 . J v, 52.35 . \mathrm{Mw}$

\section{INTRODUCTION}

The idea of the fast ignitor scheme in the inertial confinement fusion (ICF) is to pre-implode a deuterium-tritium capsule to an isochoric condition [1]. At the maximum compression point, a side of the imploded core is irradiated with a laser pulse in a few tens of a picosecond, which is much shorter than the hydrodynamic disassembly time of the irradiated spot. The petawatt (PW) -laser-generated hot electrons are supposed to increase ion temperature in the irradiation spot and initiate fusion burn.

Recently, the cone-guided PW laser enhanced neutron yield by two orders of magnitude from the imploded plasmas [2-4], and the cone guiding is investigated widely $[5,6]$. The channeling in a dense plasma by an intense laser is studied, as in $[7,8]$. Key et al. have recently reported on the electron transports related to the fast ignitor [9]. Here we report on the direct PW laser heating of a uniformly imploded deuterated-polystyrene (CD) shell plasma without coneguiding. A PW laser is synchronized to the 12 green beams from a GEKKO XII glass laser (GXII) within 10 ps [10], which implodes the spherical shell target [11]. The onedimensional hydro code (1D ILESTA) describes the implosion hydrodynamics [12].

Though the original idea of the fast ignitor might require three sets of laser pulses-for compression, channeling, and heating - we expect, nevertheless, that the PW laser plays the roles of channeling and heating at the same time, since it is as intense as self-drilling beyond the critical density and also converts its energy to hot electrons there, as predicted by the two-dimensional (2D) particle-in-cell (PIC) code [13]. The PW laser generates two temperature electrons, $200 \mathrm{keV}$ and $1 \mathrm{MeV}$. Not only the $200 \mathrm{keV}$ electrons, but also the $\mathrm{MeV}$ electrons must contribute to heating the core through collective processes, although their collisional stopping range is over the core size, which has been recently reported in Ref. [14] by Sentoku, one of the authors.

\footnotetext{
*Electronic address: yoneyosi@ile.osaka-u.ac.jp
}

The PW laser, focused to the critical density point, generates the energetic electrons within as narrow an angle as $30^{\circ}$, which heated the imploded CD shell to enhance thermal neutrons. It has not been clear when the exact timing is to heat the core. Is it just the end of the acceleration phase or after the beginning of the stagnation phase? This time, we found that the enhancement is from $(1.0 \pm 0.6) \times 10^{6} / 4 \pi$ sr to $(4.1 \pm 1.2) \times 10^{6} / 4 \pi \mathrm{sr}$ in a temporal window as $80 \pm 20 \mathrm{ps}$ in the stagnation phase. The timings agree exactly with that of $2-3 \mathrm{keV} x$-ray core emission. The bremsstrahlung from hot electrons drops simultaneously at the thermal neutron peak. These observations infer the existence of the window of effective heating by hot electrons. The PW direct illumination of the imploded sphere led us to understand the fast ignition mechanism without any conelike laser guide.

\section{EXPERIMENTAL SETUP}

The PW laser is a prepulse-free Nd:glass laser system of 0.9 petawatt peak power [10]. An optical parametric chirped amplification system is introduced to reduce the prepulses to an amplitude $<1.5 \times 10^{-8}$ that of the main pulse $[10,15]$. An energy of $190 \pm 40 \mathrm{~J}$ at $1.053 \mu \mathrm{m}$ is transported to the target with $0.6-0.7 \mathrm{ps}$ pulse length. The beam size is $50 \mathrm{~cm}$. An off-axial parabola (OAP), $21^{\circ}$ off axial and $3.8 \mathrm{~m}$ in focal length (F-number is 7.6), focuses the beam at the critical point in $15 \mu \mathrm{m}$ spot radius, $r_{0}$, providing $1 \times 10^{19} \mathrm{~W} \mathrm{~cm}^{-2}$ of the peak intensity $I_{L}$ on target. The predicted critical density point of the imploded plasma is around $200 \mu \mathrm{m}$ from the target center. It is not crucial because the Rayleigh length is $z_{R}=\pi r_{0}^{2} / \lambda \sim 670 \mu \mathrm{m}$. In the experiment, the focal point is insensitive to laser propagation in coronal plasmas, since the Rayleigh length is too long, in other words the spot size is too large. 2D-PIC indicates that such a large waist pulse would break into filaments easily and it seems that whole beam self-focusing does not happen in the current situation. Part of the front end of the chirped pulse is seeded into the preamplifier of the GXII laser. 


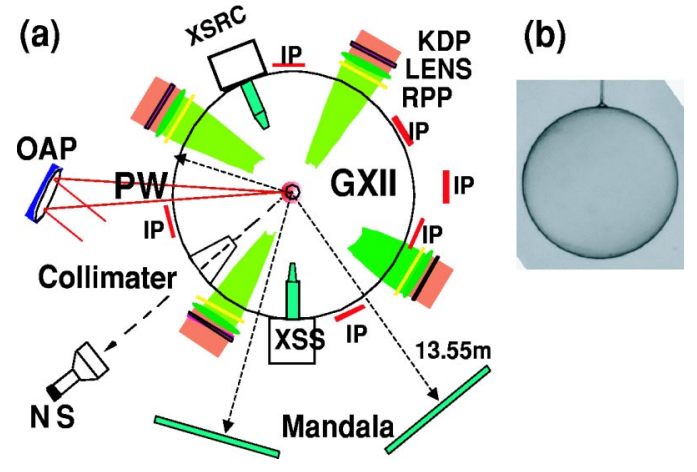

FIG. 1. (Color online) (a) Setup of PW laser, a CD shell, and GXII beams: RPP, random phase plate; OAP, off-axial parabola of F/7.6; XSRC, X-ray streak camera; XSS, X-ray streak spectrometer; and IP, imaging plate. IP is distributed on the equatorial line. Neutron plastic scintillator (NS) is at $3 \mathrm{~m}$ apart and $45^{\circ}$ backward. (b) CD shell target of $500 \mu \mathrm{m}$ in diameter and $7 \mu \mathrm{m}$ in thickness with no gas included.

To make the PW heating effect clear, the density of the compressed core is set to be more than 100 times the solid density, but its internal energy is not to exceed the PW laser power. To keep the condition, the 1D ILESTA predicted the GXII laser and target parameters as follows: Twelve $0.527 \mu \mathrm{m}$ beams are uniformly illuminated through random phase plates (RPP) tangentially onto a target, as shown in Fig. 1(a). The power is $2.34 \pm 0.45 \mathrm{~kJ}$ and the pulse is the third-order super-Gaussian in shape and $1.3 \mathrm{~ns}$ in width full width at half maximum. Energy balance between the beams is $93 \pm 3 \%$. Targets are $501 \pm 12 \mu \mathrm{m}$ in diameter and $6.9 \pm 0.62 \mu \mathrm{m}$ in thickness. No gas is filled. Figure 1(b) is the photograph.

The PW-laser-generated hot electrons hit the chamber wall and emit the bremsstrahlung. The chamber is $1.6 \mathrm{~m}$ in diameter and the wall is made of stainless steel of $8 \mathrm{~cm}$ thickness.

As seen in Fig. 1(a), the imaging plates IP(Fuji BASSR2025) detected the angular distribution of the emission on the equator of the outer surface of the target chamber. The sensitivity to electron decreases as increasing beam energy until $11.5 \mathrm{MeV}$, at which point it is almost flat to 0.007 photostimulated luminescence (PSL)/electron $\pm 10 \%$ up to $100 \mathrm{MeV}$ [16]. PSL is a relative IP unit after photostimulated luminescence.

D-D neutrons are detected by a fast plastic (BC-422) scintillator (NS) of $10 \mathrm{~cm}$ diameter and $5 \mathrm{~cm}$ length, set at $3.0 \mathrm{~m}$ from the target and $43^{\circ}$ back to the PW laser axis. A collimator, shown in Fig. 1(a), is made with lead and plastics to suppress the neutrons scattered from the chamber wall [17]. The solid angle of detection is $8.7 \times 10^{-4} \mathrm{sr}$. Mandala, consisting of two panels, located $13.55 \mathrm{~m}$ far from the target is used to calibrate the yield of the plastic scintillator $[18,19]$. One panel consists of a 421-channel array of single-hit scintillation detector.

By fitting the $\mathrm{x}$-ray streak trace of the ablation surface to the 1D ILESTA, we determined the onset of the stagnation, that is, the timing when the first shock bounces back to the imploding shell, as shown in the flow lines in Fig. 2(a).
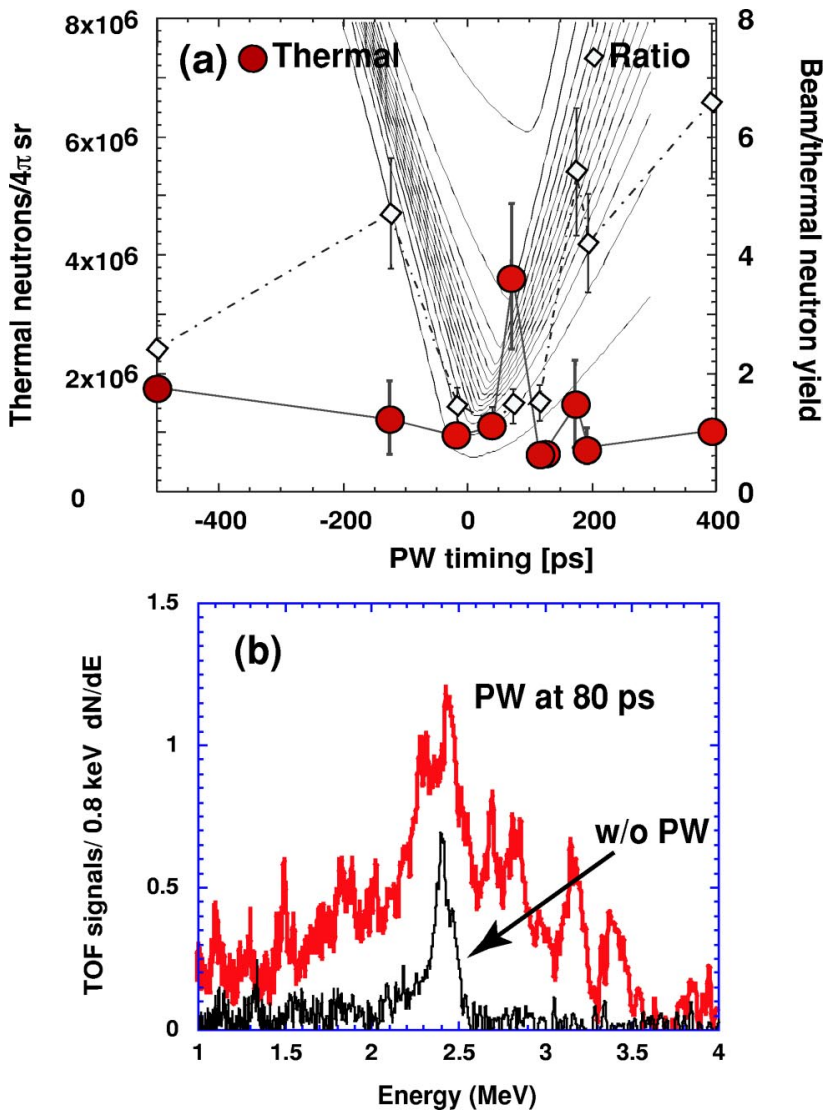

FIG. 2. (Color online) (a) Thermal neutron yield for $4 \pi$ sr (dot) from NS and the ratio of beam fusion neutrons $(1.5$ to $3.5 \mathrm{MeV})$ to thermal neutrons $(2.45 \pm 0.2 \mathrm{MeV})$ (diamond) against PW timing. Flow lines: 1D ILESTA shell radius $(0-60 \mu \mathrm{m})$. Horizontal error is \pm 40 ps. (b) DD neutron signals against neutron energy. Upper: with PW of $187 \mathrm{~J}$ on target at $+80 \mathrm{ps}$; lower: without PW laser.

Typically, it is 800 ps after the GXII laser peak. The simultaneous shot of PW and GXII on a glass rod provided us a timing accuracy within $\pm 20 \mathrm{ps}$, including the streak camera reading error.

\section{FLOW DIAGRAM AND NEUTRON ENHANCEMENT}

Figure 2(a) shows the change of thermal neutron yield $(2.45 \pm 0.2 \mathrm{MeV})$ (dot) against PW timing. In as narrow a window as from 60 to $100 \mathrm{ps}$, the thermal neutron is enhanced from $(1.0 \pm 0.6) \times 10^{6} / 4 \pi$ sr to $(4.1 \pm 1.2)$ $\times 10^{6} / 4 \pi$ sr. The enhancement of yield is four to five times. The PW laser energy is less than $10 \%$ of the implosion laser energy. However, since the internal energy of the imploded core plasma is simulated to be about $50 \mathrm{~J}$, the PW laser will increase the core temperature, resulting in the neutron increment. In the previous cone target case, the neutron yield without PW laser has been as low as an order of $10^{4}$ due to nine-beam implosion [3]. The resulting enhancement of the neutron is 100 times or more, even though the yield itself is the same as the present case, probably because the PW energy is the same for both cases. 


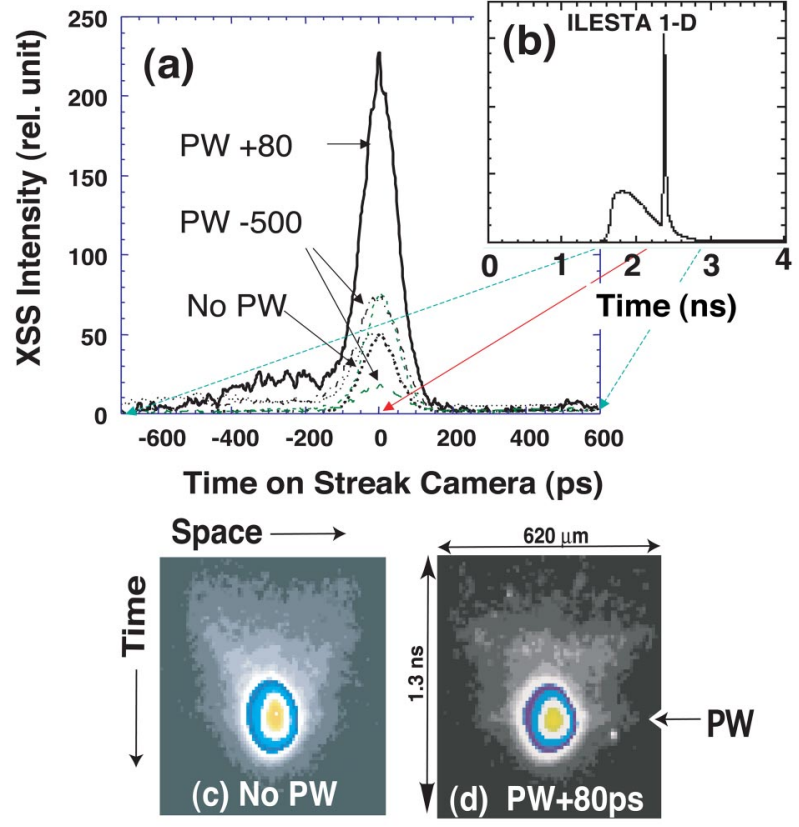

FIG. 3. (Color online) (a) Time history of x-ray core emission from XSS with PW (bold line) and without or with but at -500 ps (dashed and dotted lines). Peak intensity at compression is plotted in Fig. 5. Timing origin is relative. (b) $0.05-1.52 \mathrm{keV}$ x-ray emissions calculated by 1D ILESTA. Peak is at 100 ps after first shock bounce. (c) X-ray streak image without PW laser and (d) with PW at +80 ps. Full scale: $1.3 \mathrm{~ns} \times 620 \mu \mathrm{m}$.

No thermal enhancement occurs around 100 ps. The second small peak appears between 160 and 200 ps. Figure 2(b) compares the neutron signal with the $\mathrm{PW}$ at $80 \mathrm{ps}$ to the signal without PW. Here the original time-of-flight signals are plotted as a function of the neutron kinetic energy. The Gaussian shape fitting to the signals provides the ion temperatures $T_{i}$ to be $0.81 \mathrm{keV}$ without PW laser and $1.2 \mathrm{keV}$ with it. Since the 1D ILESTA shows us that the neutron yield increases in proportion to the fifth power of the ion temperature, the yield multiplication may be $(1.2 / 0.81)^{5} \sim 7$. This is close to the maximum enhancement, $\sim 5$.

Although the enhanced temperature might be somewhat high, this may be because the beam fusion component is mixed as well as high gamma-ray noise. The background flow lines in (a) are from the 1D ILESTA, which predicts the stagnation phase is from 0 to $100 \mathrm{ps}$.

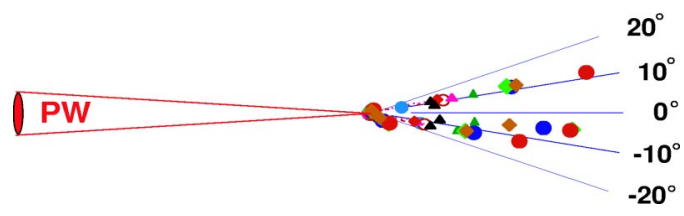

FIG. 4. (Color online) Bremsstrahlung focuses in a cone angle of $30^{\circ}$ around the PW axis. Ten different marks correspond to ten different shots. No data on $0^{\circ}$ axis, because of a plastic window.
The 2D PIC predicts that $90 \%$ of PW energy is converted to hot electrons, but $4 \%$ goes to deuterons, the temperature of which is $40 \mathrm{keV}$. They collide with cold deuterons in the peripheral plasmas, generating beam fusion neutrons over the range from 2 to a few $\mathrm{MeV}$. So produced neutrons appear as many peaks on both sides of the thermal peak in (b) [20]. We plotted in (a) the ratio of beam fusion neutron yield (from 1.5 to $3.5 \mathrm{MeV}$ area) to the thermal yield by diamond. Note that, although the PW laser produces beam fusion neutrons at either timing of the acceleration, stagnation, and disruption phases, the results show that the ratio reduces to about 1.5 only from 100 to 160 ps within the stagnation. Otherwise it becomes larger than three times and attains seven times at $400 \mathrm{ps}$, where the ion energy spread is over $40 \mathrm{keV}$. The counts for Mandala, though at the perpendicular direction, are too low to define the temperature.

\section{CORE HEATING AND X-RAY EMISSION ENHANCEMENT}

To make clear whether the PW laser heats the core plasma or only the peripheral ones, we checked the X-ray core emission using an X-ray streak spectrometer (XSS) [21], which is a Bragg crystal spectrometer coupled with a compact X-ray streak camera of $2-3 \mathrm{keV}$. The XSS peak is typically seen at 100 ps after the first shock bouncing point, as predicted by the 1D ILESTA in (b) and as discussed in Ref. [21]. Figure 3(a) shows that the XSS peak is strongly enhanced when the PW laser is adjusted at the timing of neutron increment. Otherwise, no enhancement is observed, either with or without the PW laser (thin and dashed lines). As indicated by an arrow in (d), the XSS peak is also seen as a sharp horizontal line in the $\mathrm{x}$-ray streak image of the core implosion, which would be enhanced by the PW.

\section{ANGULAR DISTRIBUTION OF HOT ELECTRONS}

The angular distribution of the hot electron emissions is much narrower that expected. Figure 4 shows that the angu-

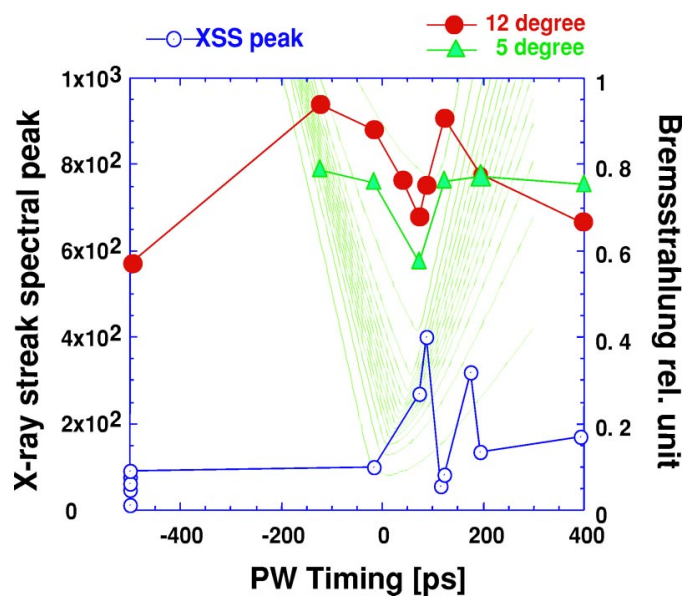

FIG. 5. (Color online) Two XSS peaks (open circle) at 80 and 180 ps agree with the neutrons' peaks in Fig. 2(a). Bremsstrahlung (dot: $12^{\circ}$ to the PW axis and triangle: $5^{\circ}$ ) have a sharp dip at the first neutron peak. 

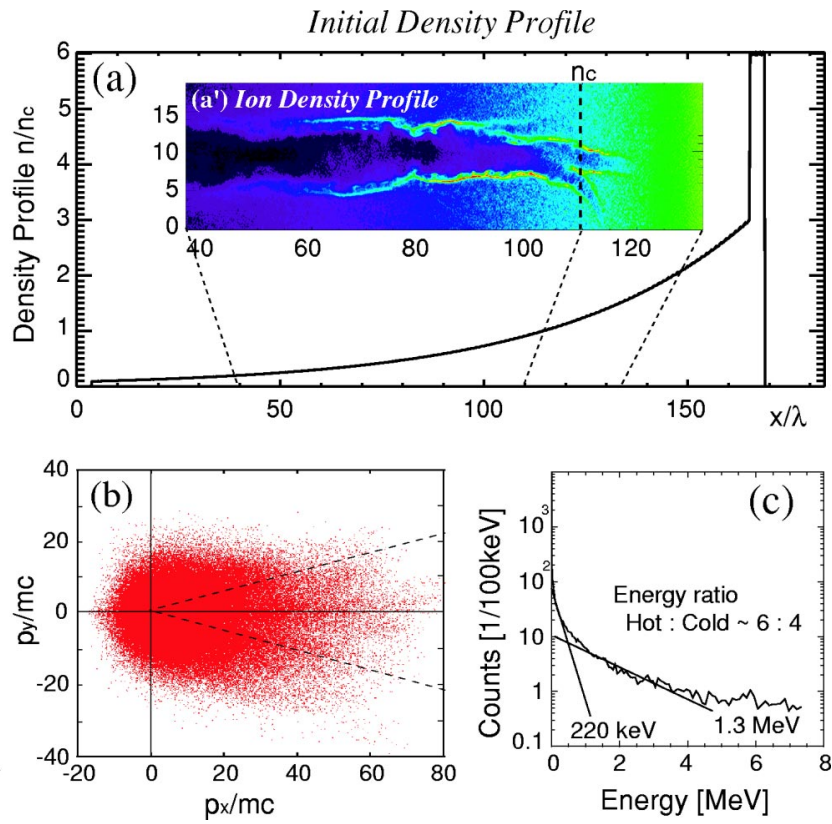

FIG. 6. (Color online) (a) The ion density profile around the critical density used in the 2D PIC code; (a') ion density profile snowplowed by a laser at $700 \mathrm{fs}$, which penetrates $10 \mu \mathrm{m}$ into the overdense region. (b) Electron phase map from the 2D PIC code at 700 ps. (c) Hot electron energy spectrum in the cone. Hot electron temperature is $1.3 \mathrm{MeV}$ and cold electron temperature is $220 \mathrm{keV}$. Energy ratio of hot to cold is 6:4. $x, y$ directions are PW axial and transverse ones, respectively.

lar divergence of the IP counts is $30^{\circ}$ around the laser axis, in agreement with the PIC result, as discussed later. Though the IPs on the stainless steel wall record the counts, an IP on the 1-cm-thick plastic window of the counter port to the PW laser shows only a count of $10^{-2}$ of the other IPs. The hot electrons from the target plasma would reach the chamber wall and produce the bremsstrahlung emissions, which then hit IPs. Consequently, the angular distribution seems to reflect the angular distribution of the hot electron emission. The 2D PIC predicts the hot electron temperature to be $1.3 \mathrm{MeV}$ and collimation of very energetic electrons to the forward direction (see Fig. 6). The emission is observed also to the backward direction, but the intensity is only $2 \times 10^{-4}$ of the forward one.

In Fig. 5, two peaks of the XSS (open circles at 80 and $180 \mathrm{ps}$ ) agree exactly with those of the thermal neutrons in Fig. 2(a), which may lead to the PW laser heating the core plasma so that it emits $2-3 \mathrm{keV}$ emissions.

Also in Fig. 5, we plotted the forward bremsstrahlung (small dot at $12^{\circ}$ to the PW axis and small triangle at $5^{\circ}$, both of which have a sharp dip at the first neutron peak). The sharp dip seems to show that the core plasma at the maximum compression absorbs about $30 \%$ of hot electrons. Taking the neutron enhancement 4, we imply that the ion temperature increases $1.3\left(=4^{1 / 5}\right)$ times. The 1D ILESTA gives that the imploding laser GXII delivers the energy of $\sim 50 \mathrm{~J}$ to the core plasma without PW. Therefore, the energy deposition from $\mathrm{PW}$ in the core is estimated as $50 \mathrm{~J}$ $\times 0.3=15 \mathrm{~J}$, which corresponds to about $40 \%$ of PW laser energy. This agrees with the observation that the dip of the bremsstrahlung corresponds to $30 \%$ absorption of the hot electrons, or $30 \% \times 90 \%$ PW laser, as seen in the $2 \mathrm{D}-\mathrm{PIC}$ calculation.

\section{2D-PIC PREDICTION OF PW LASER PENETRATION AND GENERATION OF BOTH ENERGETIC ELECTRONS AND IONS}

Using an exponential ion density profile provided by the 1D ILESTA as shown in Fig. 6(a), the 2D PIC [13] was performed to predict the PW laser penetration into the critical density plasma and generation of both energetic electrons and ions. The laser is coming from the left boundary with $2 \times 10^{19} \mathrm{~W} \mathrm{~cm}^{-2}$ intensity. The pulse length and spot size are $275 \mathrm{fs}$ and $10 \mu \mathrm{m}$, respectively. Figure $6\left(\mathrm{a}^{\prime}\right)$ is the ion density profile at $400 \mathrm{fs}$ after the laser pulse switching off, which shows that the laser snowplows the plasma to the radial direction and penetrates $10 \mu \mathrm{m}$ into the overdense region. Hot electrons are generated around the critical point, whose phase map is shown in (b). Although the electrons of lower than a few MeV whose temperature is $220 \mathrm{keV}$ are uniformly distributed, those of higher energy are directed forward into a $30^{\circ}$ cone, which agrees with the bremsstrahlung in Fig. 5. The hot electron spectrum in the $30^{\circ}$ cone is shown in (c). Here, about $60 \%$ of the electron energy is in hot electrons, of which temperature is $1.3 \mathrm{MeV}$.

By using the electron and ion phase maps, we estimated heating of the imploded overdense plasma. The hot electron penetrates into the central dense region and may heat the core, although such energetic electrons' classical stopping range is over a millimeter. Electrons flowing into the overdense plasma excite magnetic perturbations, which will induce a strong anomalous resistivity. From the scaling of stopping range by this anomalous process in Ref. [14], a $1 \mathrm{MeV}$ electron will be stopped at $30 \mu \mathrm{m}$ in the compressed core, where its peak density is $\sim 10^{25} \mathrm{~cm}^{-3}$. Hence, those collimated highly energetic electrons can contribute to heating the core. Although the accelerated deuterons heat only the peripheral plasmas under $10^{23} \mathrm{~cm}^{-3}$, resulting in beam fusion reaction, the hot electrons penetrate into the central dense region and heat the core. Since the neutron yield is proportional to the square of the density and the core density is much higher than the peripheral region, the thermal fusion dominates in the core region.

\section{CONCLUSION}

The PW laser, focused to the critical density point, generates the energetic electrons within as narrow an angle as $30^{\circ}$, which heated the imploded CD shell to enhance thermal neutrons. The enhancement is from $(1.0 \pm 0.6) \times 10^{6} / 4 \pi \mathrm{sr}$ to $(4.1 \pm 1.2) \times 10^{6} / 4 \pi \mathrm{sr}$ in a temporal window as $80 \pm 20 \mathrm{ps}$ in the stagnation phase. The timings exactly agree with that of 2-3 keV x-ray core emission. The bremsstrahlung from hot electrons drops simultaneously at the thermal neutron peak. 
These observations infer the existence of the window of effective heating by hot electrons.

The PW directly heated the imploded sphere without any conelike laser guide. Thus the fast ignitor mechanism is understood.

\section{ACKNOWLEDGMENTS}

The authors would like to thank H. Fujita, H. Yoshida, S. Matsuo, T. Kawasaki, and PW laser members as well as PEII, GOD, and MT members for their technical support, and all the ILE members for their encouragement.
[1] Max Tabak et al., Phys. Plasmas 1, 1626 (1994).

[2] R. Kodama et al., Nature (London) 412, 798-802 (2001).

[3] R. Kodama et al., Nature (London) 418, 933-934 (2002).

[4] P. A. Norreys et al., Phys. Plasmas 11, 2746 (2004).

[5] R. B. Stephens et al., Phys. Rev. Lett. 91, 185001 (2003).

[6] Y. Sentoku et al., Phys. Plasmas 11, 3083 (2004).

[7] Z. Najmudin et al., Phys. Plasmas 10, 438 (2003).

[8] T. Matsuoka, Ph.D. thesis, Osaka University, 2004.

[9] M. H. Key et al., in Proceedings on Inertial Fusion Science and Applications 2003, edited by B. A. Hammel, D. D. Meyerhofer, J. Meyer-ter-Vehn, and H. Azechi, (American Nuclear Society, Inc., Illinois, 2004), p. 353.

[10] Y. Kitagawa et al., IEEE J. Quantum Electron. 40, 281 (2004).
[11] H. Azechi et al., Laser Part. Beams 9, 193 (1991).

[12] H. Takabe et al., Phys. Fluids 31, 2884 (1988).

[13] Y. Sentoku et al., Phys. Plasmas 5, 4366 (1998).

[14] Y. Sentoku et al., Phys. Rev. Lett. 90, 155001 (2003).

[15] H. Yoshida et al., Opt. Lett. 28, 257 (2003).

[16] T. Takahashi et al., Ionizing Radiation 28, 203 (2002).

[17] H. Habara et al., Phys. Plasmas 10, 3712 (2003).

[18] N. Izumi et al., Rev. Sci. Instrum. 70, 1221 (1999).

[19] N. Izumi et al., Phys. Rev. E 65, 036413 (2002).

[20] Y. Kitagawa et al., Phys. Plasmas 9, 2202 (2002).

[21] K. Fujita et al., J. Quant. Spectrosc. Radiat. Transf. 58, 585 (1997). 